\title{
Sporadic Cancer
}

National Cancer Institute ( $\mathrm{NCl})$

\section{Source}

National Cancer Institute (NCI). Sporadic Cancer.

This term has two meanings. It is sometimes used to differentiate cancers occurring in people who do not have a germline mutation that confers increased susceptibility to cancer from cancers occurring in people who are known to carry a mutation. Cancer developing in people who do not carry a high-risk mutation is referred to as sporadic cancer. The distinction is not absolute, because genetic background may influence the likelihood of cancer even in the absence of a specific predisposing mutation. Alternatively, sporadic is also sometimes used to describe cancer occurring in individuals without a family history of cancer. 